\title{
Tissue metabolomic fingerprinting reveals metabolic disorders associated with human gastric cancer morbidity
}

\author{
HU SONG ${ }^{1}$, LEI WANG ${ }^{2}$, HUAN-LIANG LIU ${ }^{2}$, XIAO-BIN WU ${ }^{1}$, HUA-SHE WANG ${ }^{1}$, ZHONG-HUI LIU $^{1}$, \\ YUN LI $^{1}$, DE-CHANG DIAO ${ }^{1}$, HONG-LEI CHEN $^{1}$ and JUN-SHENG PENG ${ }^{1}$ \\ ${ }^{1}$ Department of Gastrointestinal Surgery, The Sixth Affiliated Hospital (Gastrointestinal and Anal Hospital), \\ ${ }^{2}$ Institute of Gastroenterology, Sun Yat-sen University, Guangzhou, Guangdong 510655, P.R. China
}

Received February 3, 2011; Accepted April 1, 2011

DOI: $10.3892 / o r .2011 .1302$

\begin{abstract}
The principal way to improve the outcome of gastric cancer (GC) is to predict carcinogenesis and metastasis at an early stage. The aims of the present study were to test the hypothesis that distinct metabolic profiles are reflected in GC tissues and to further explore potential biomarkers for GC diagnosis. Gas chromatography/mass spectrometry (GC/MS) was utilized to analyze tissue metabolites from $30 \mathrm{GC}$ patients. A diagnostic model for GC was constructed using orthogonal partial least squares discriminant analysis (OPLS-DA), and the metabolomic data were analyzed using the non-parametric Wilcoxon rank sum test to identify the metabolic tissue biomarkers for GC. Over 100 signals were routinely detected in one single total ion current (TIC) chromatogram, and the OPLS-DA model generated from the metabolic profile of the tissues adequately discriminated the GC tissues from the normal mucosae. Among the low-molecular-weight endogenous metabolites, a total of 41 compounds, such as amino acids, organic acids, carbohydrates, fatty acids and steroids, were detected, and 15 differential metabolites were identified with significant difference $(\mathrm{p}<0.05)$. A total of 20 variables were noted which contributed to a great extent in the discriminating OPLS-DA model (VIP value >1.0), among which 12 metabolites were identified using both VIP values (VIP $>1$ ) and the Wilcoxon test $(\mathrm{p}<0.05)$. In conclusion, the identification
\end{abstract}

Correspondence to: Dr Jun-Sheng Peng, Department of Gastrointestinal Surgery, The Sixth Affiliated Hospital (Gastrointestinal and Anal Hospital), Sun Yat-sen University, 26 Yuan Cun Er Heng Road, Guangzhou, Guangdong 510655, P.R. China

E-mail: pengjunsheng@tom.com

Abbreviations: GC, gastric cancer; GC/MS, gas chromatography/ mass spectrometry; EA, ethyl acetate; BSTFA, N,O-Bis (trimethylsilyl) trifluoroacetamide; MS, mass spectrometry; TIC, total ion current; OPLS-DA, orthogonal partial least squares discriminant analysis; TNM, tumor-node-metastasis; TCA, tricarboxylic acid; FC, fold change

Key words: metabonomics, metabolic perturbations, gastric carcinoma of the metabolites associated with GC morbidity potentially revealed perturbations of glycolysis, fatty acid $\beta$-oxidation, cholesterol and amino acid metabolism. These results suggest that tissue metabolic profiles have great potential in detecting GC and may aid in understanding its underlying mechanisms.

\section{Introduction}

Gastric cancer (GC) is one of the most prevalent and deadly forms of cancers worldwide, with nearly a million new cases diagnosed each year. It is the fourth most common type of cancer and the second most frequent cause of cancer-related mortality in the world $(1,2)$. The relatively asymptomatic nature in the early stage of the disease and the lack of adequate screening methods have resulted in the majority of GC patients diagnosed at an advanced stage of the disease. The prognosis of patients with advanced cancer still remains poor due to its high recurrence rate and metastatic features including lymph node invasion, peritoneal implantation and liver metastasis (3-5). Therefore, the most effective way to improve patient prognosis is the prediction of GC carcinogenesis and metastasis at an early stage. Currently, endoscopy is widely used for early screening but this methodology involves invasive procedures and its cost effectiveness remains disputable. Thus, a full understanding of the molecular mechanism of carcinogenesis and identification of biomarkers for the early diagnosis and effective treatment of human GC are crucial. Although several serum biomarkers have been researched as new invasive tools for screening GC (6-9), these serum tests are not available as screening or surveillance tests due to their low specificity and sensitivity (9). Biomarkers derived from tumor tissues may provide higher sensitivity and specificity than those from serum (10), yet tissue specimens must be obtained by invasive means involving endoscopy and biopsy. Tissue metabolic profiling offers the opportunity to understand the molecular mechanism involved in carcinogenesis and to aid in the identification of potential biomarkers for the early diagnosis and effective treatment of human GC.

Metabolomics is a recently developed technology for detection, identification and quantification of low-molecularweight metabolites that are involved in the metabolism in an organism at a specified time under specific environmental conditions $(11,12)$. This technique is an effective tool for 
disease diagnosis (13-15), biomarker screening (16-20) and characterization of biological pathways (11). Recent technological advances in NMR spectroscopy and mass spectrometry have further improved the sensitivity and spectral resolution of cancer metabolomic study (21). Among the various techniques conventionally used for cancer metabolic profiling, gas chromatography/mass spectrometry (GC/MS) has been proven to be a robust metabolomic tool and is widely applied in metabolite identification and quantification based on its high sensitivity, peak resolution and reproducibility (22-24). Since cancers are known to possess highly unique metabolic phenotypes, identification of specific biomarkers using metabolomics may be useful for early cancer detection and prognosis. 'Metabolic profiling' has been proposed as a means of measuring the total complement of individual metabolites in a given biological sample. The physiological state of a complex tissue is reflected in the full complement of various metabolites by its constituent cells. Hence, metabolic profiling may offer a unique opportunity for the understanding of the molecular mechanisms involved.

In the present study, we hypothesized that the comprehensive analysis of tissue metabolites defines metabolic signatures that discriminate GC specimens from normal specimens. To test this hypothesis, we utilized GC/MS metabonomics to fingerprint tumor tissues and their matched normal mucosae. Our study demonstrated the metabolic perturbations of GC patients and contributed to the identification of potential metabolic biomarkers associated with GC morbidity. This technique is a potential alternative non-invasive means for GC detection.

\section{Materials and methods}

Chemicals and reagents. Pyridine and N,O-bis(trimethylsilyl) trifluoroacetamide (BSTFA) were purchased from SigmaAldrich (St. Louis, MO). Chromatogram-grade methanol, ethyl acetate and chloroform were purchased from Sijia Chemicals Group Corp. (Guangzhou, China). Reference standards of chromatogram grade such as methionine, phenylalanine, proline, lysine, tyrosine, glutamate, lauric acid, palmitic acid, stearic acid, oleic acid, arachidonic acid, glucose and cholesterol were also purchased from Sijia Chemicals Group Corp.

Patient recruitment and sample collection. The study protocol was approved by the Ethics Committee of The Sixth Affiliated Hospital, Sun Yat-sen University, and informed consent was obtained from each participant prior to sample collection. Gastric tissues including tumor and adjacent matched normal mucosa from 30 patients were collected from freshly isolated resections carried out at The Sixth Affiliated Hospital, Sun Yat-sen University. All of the patients including 15 males $(50 \%)$ and 15 females $(50 \%)$ were of Chinese ethnic origin, and the patient age ranged from 39 to 88 years. None of the patients were diagnosed with metabolic diseases such as diabetes mellitus or hyperthyroidism. The tissue specimens were dissected by an experienced gastric surgeon, and adjacent normal mucosae were obtained at least $8 \mathrm{~cm}$ from the tumor margin. Histological differentiation, tumor stage (25) and resection margin were confirmed using routine histopatho- logical H\&E-stained specimens. The anatomical site, tissue histology, tumor grade and TNM stages are presented in Table I. The specimens were carefully cleaned in saline water and immediately snap-frozen in liquid nitrogen within $30 \mathrm{~min}$, labeled and stored at $-80^{\circ} \mathrm{C}$ until processing.

Specimen preparation for GC/MS analysis. Fifty milligrams of each tissue was transferred to 2.0-ml Eppendorf tubes and lyophilized and extracted with $1.0 \mathrm{ml}$ of a mixture of methanol/ chloroform (3:1, v/v) and vortexed for $30 \mathrm{sec}$ (26). After storing for $10 \mathrm{~min}$ at $-20^{\circ} \mathrm{C}$, the samples were subsequently centrifuged at $12,000 \mathrm{x} \mathrm{g}$ for $10 \mathrm{~min}$ at $4^{\circ} \mathrm{C}$, and $200 \mu \mathrm{l}$ of the supernatant was collected separately from each sample and transferred to a 1.5-ml Eppendorf tube. The collected supernatant was concentrated to complete dryness using liquid nitrogen and maintained at $-30^{\circ} \mathrm{C}$ until analysis.

A total of $50 \mu \mathrm{l}$ ethyl acetate (EA) was added to each of the dried tissue extracts, vortex-mixed for $1 \mathrm{~min}$, and then $100 \mu \mathrm{l}$ derivatization reagent, a mixture of BSTFA, pyridine and EA $(3: 1: 1, \mathrm{v} / \mathrm{v} / \mathrm{v})$, was added to the extract for derivatization for $16 \mathrm{~h}$ at room temperature (27). The resulting solution was then vortex for $1 \mathrm{~min}$ and transferred to an amber glass vial for $\mathrm{GC} / \mathrm{MS}$ analysis. In order to prevent a batch effect all assays were conducted at random in a double blinded manner.

One milligram of amino acids (methionine, phenylalanine, proline, lysine, tyrosine and glutamate), fatty acids (lauric acid, palmitic acid, stearic acid, oleic acid, arachidonic acid), glucose and cholesterol reference standard was dissolved in $5 \mathrm{ml}$ of pyridine to produce a final concentration of $200 \mu \mathrm{g} /$ $\mathrm{ml}$. Each $200 \mu \mathrm{l}$ of reference standard was transferred to 2.0-ml Eppendorf tubes and derivatized with the procedure illustrated above and prepared for GC/MS analysis according to the methodology described below.

GC/MS analysis. GC/MS analysis was performed using an Agilent 7890A Gas Chromatography system equipped with an Agilent DB-5MS capillary column (30x0.25 mm ID, $0.25 \mu \mathrm{m}$ film thickness) with an Agilent 5975C Series autosampler (Agilent Technologies). Each $1 \mu \mathrm{l}$ aliquot of the derivatized solution or derivatized reference standard was injected in splitless mode, and helium was used as the carrier gas at a constant flow rate of $1.0 \mathrm{ml} / \mathrm{min}$. The temperature of the inlet, transfer line, ion source and quadrupole was maintained at $270,260,200$ and $150^{\circ} \mathrm{C}$, respectively. The oven temperature programming was set to 2 -min isothermal heating at $80^{\circ} \mathrm{C}$, followed by $10^{\circ} \mathrm{C} / \mathrm{min}$ oven temperature ramps to $180^{\circ} \mathrm{C}, 5^{\circ} \mathrm{C} /$ min to $240^{\circ} \mathrm{C}$, and $10^{\circ} \mathrm{C} / \mathrm{min}$ to $260^{\circ} \mathrm{C}$, and a final 8 -min maintenance at $260^{\circ} \mathrm{C}$. The mass spectrometry (MS) was operated at electron impact (EI) ionization mode at $70 \mathrm{eV}$. Data acquisition was achieved using MS in an electron impact mode and full scan monitoring mode from $\mathrm{m} / \mathrm{z} 30$ to 600 with an acquisition rate of $20 \mathrm{spectra} / \mathrm{sec}$. Solvent delay time was set at $5 \mathrm{~min}$.

Data processing for GC/MS and statistical analysis. After GC/MS analysis, each sample or reference standard was represented by a GC/MS total ion current (TIC) chromatogram. Among the detected peaks, a multi-dimensional vector was constructed manually to characterize the compounds (27-29). Each vector was normalized to the total sum of the vector 
Table I. Summary of the anatomical and clinicopathological characteristics of the GC patients.

\begin{tabular}{|c|c|c|c|c|c|c|c|c|}
\hline $\begin{array}{l}\text { Patient } \\
\text { no. }\end{array}$ & Age & Gender & Race & $\begin{array}{l}\text { Anatomic } \\
\text { site }\end{array}$ & Histology & $\begin{array}{l}\text { Depth of } \\
\text { invasion }\end{array}$ & $\begin{array}{l}\text { Lymph node } \\
\text { metastasis }\end{array}$ & $\begin{array}{l}\text { TNM } \\
\text { stage }\end{array}$ \\
\hline 1 & 61 & $\mathrm{M}$ & Chinese & Antrum & WDA & $\mathrm{T} 1$ & No & IA \\
\hline 2 & 56 & $\mathrm{M}$ & Chinese & Body & PDA & $\mathrm{T} 1$ & No & IA \\
\hline 3 & 42 & $\mathrm{M}$ & Chinese & Body & PDA & $\mathrm{T} 2$ & No & IB \\
\hline 4 & 65 & M & Chinese & Body & PDA & $\mathrm{T} 2$ & No & IB \\
\hline 5 & 65 & $\mathrm{M}$ & Chinese & Gastric stump & MDA & $\mathrm{T} 3$ & No & IIA \\
\hline 6 & 72 & M & Chinese & Antrum & MDA & $\mathrm{T} 3$ & No & IIA \\
\hline 7 & 76 & M & Chinese & Body & MDA & $\mathrm{T} 3$ & No & IIA \\
\hline 8 & 72 & $\mathrm{~F}$ & Chinese & Antrum & PDA & $\mathrm{T} 3$ & No & IIA \\
\hline 9 & 77 & $\mathrm{~F}$ & Chinese & Body & WDA & $\mathrm{T} 3$ & No & IIA \\
\hline 10 & 66 & $\mathrm{M}$ & Chinese & Antrum & PDA & $\mathrm{T} 3$ & $\mathrm{~N} 1$ & IIIA \\
\hline 11 & 78 & $\mathrm{M}$ & Chinese & Antrum & PDA & $\mathrm{T} 3$ & $\mathrm{~N} 2$ & IIIB \\
\hline 12 & 63 & M & Chinese & Body & PDA & $\mathrm{T} 3$ & $\mathrm{~N} 2$ & IIIB \\
\hline 13 & 62 & $\mathrm{M}$ & Chinese & Body & PDA & $\mathrm{T} 3$ & N2 & IIIB \\
\hline 14 & 61 & $\mathrm{M}$ & Chinese & Fundus & PDA & $\mathrm{T} 3$ & $\mathrm{~N} 1$ & IIIA \\
\hline 15 & 47 & $\mathrm{M}$ & Chinese & Body & PDA & $\mathrm{T} 3$ & N1 & IIIA \\
\hline 16 & 55 & $\mathrm{M}$ & Chinese & Body & PDA & $\mathrm{T} 3$ & $\mathrm{~N} 2$ & IIIB \\
\hline 17 & 47 & $\mathrm{~F}$ & Chinese & Antrum & PDA & $\mathrm{T} 3$ & $\mathrm{~N} 2$ & IIIB \\
\hline 18 & 42 & $\mathrm{~F}$ & Chinese & Stomach angle & MDA & $\mathrm{T} 3$ & N1 & IIIA \\
\hline 19 & 40 & $\mathrm{~F}$ & Chinese & Antrum & PDA & $\mathrm{T} 3$ & $\mathrm{~N} 2$ & IIIB \\
\hline 20 & 82 & $\mathrm{~F}$ & Chinese & Antrum & PDA & $\mathrm{T} 3$ & N1 & IIIA \\
\hline 21 & 51 & $\mathrm{~F}$ & Chinese & Body & PDA & $\mathrm{T} 3$ & N1 & IIIA \\
\hline 22 & 69 & $\mathrm{~F}$ & Chinese & Antrum & PDA & $\mathrm{T} 3$ & N1 & IIIA \\
\hline 23 & 39 & $\mathrm{~F}$ & Chinese & Body & PDA & $\mathrm{T} 3$ & $\mathrm{~N} 2$ & IIIB \\
\hline 24 & 77 & $\mathrm{~F}$ & Chinese & Body & PDA & $\mathrm{T} 3$ & $\mathrm{~N} 1$ & IIIA \\
\hline 25 & 61 & M & Chinese & Body & MDA & $\mathrm{T} 4$ & $\mathrm{~N} 1$ & IV \\
\hline 26 & 56 & $\mathrm{~F}$ & Chinese & Antrum & PDA & $\mathrm{T} 3$ & $\mathrm{~N} 3$ & IV \\
\hline 27 & 61 & $\mathrm{~F}$ & Chinese & Pylorus & PDA & $\mathrm{T} 4$ & N3 & IV \\
\hline 28 & 47 & $\mathrm{~F}$ & Chinese & Body & PDA & $\mathrm{T} 3$ & N3 & IV \\
\hline 29 & 88 & $\mathrm{~F}$ & Chinese & Cardia & PDA & $\mathrm{T} 3$ & N3 & IV \\
\hline 30 & 66 & $\mathrm{~F}$ & Chinese & Cardia & PDA & $\mathrm{T} 3$ & $\mathrm{~N} 3$ & IV \\
\hline
\end{tabular}

Age, in years. M, male; F, female; PDA, poorly differentiated adenocarcinoma; MDA, moderately differentiated adenocarcinoma; WDA, well differentiated adenocarcinoma.

intensities (27,28), and peaks attributed to column bleed and the derivatization reagent were removed. Those peaks were taken into account provided they were consistently detected in at least $80 \%$ of samples (30). All of the detected peaks were identified by comparing both the MS spectra and the retention index (RI) (31) with those available in the National Institute of Standards and Technology (NIST) mass spectral library (Wiley Registry, 9th edition, 2008) and customized reference mass spectral libraries (32). The mass spectra obtained were investigated carefully, and only those molecules with matching probability $>80 \%$ were considered. Within each sample, the retention time (RT) and m/z data pairs were used as the identifier for each peak, and the ion intensities for each peak detected were then normalized to the sum of the peak intensities in that sample. To account for any difference in concentration between samples, all data were normalized to a total value of 100 .

Normalized data were exported to SIMCA-P+ (version 11.5, Umetrics, Umeå, Sweden) to perform orthogonal partial least squares discriminant analysis (OPLS-DA) where grouping trends and outliers in the data were observed and a model was constructed to identify marker metabolites that differentiated GC and non-GC cohorts $(33,34)$. From the normalized data, an indication of statistical significance was based on a non-parametric two-tailed paired Wilcoxon analysis performed with SPSS 13.0 for Windows (SPSS, Chicago, IL). P-values of $<0.05$ were considered as statistically significant. The fold change (FC) was based on the mean value of $30 \mathrm{GC}$ specimens relative to the mean of non-GC specimens. 

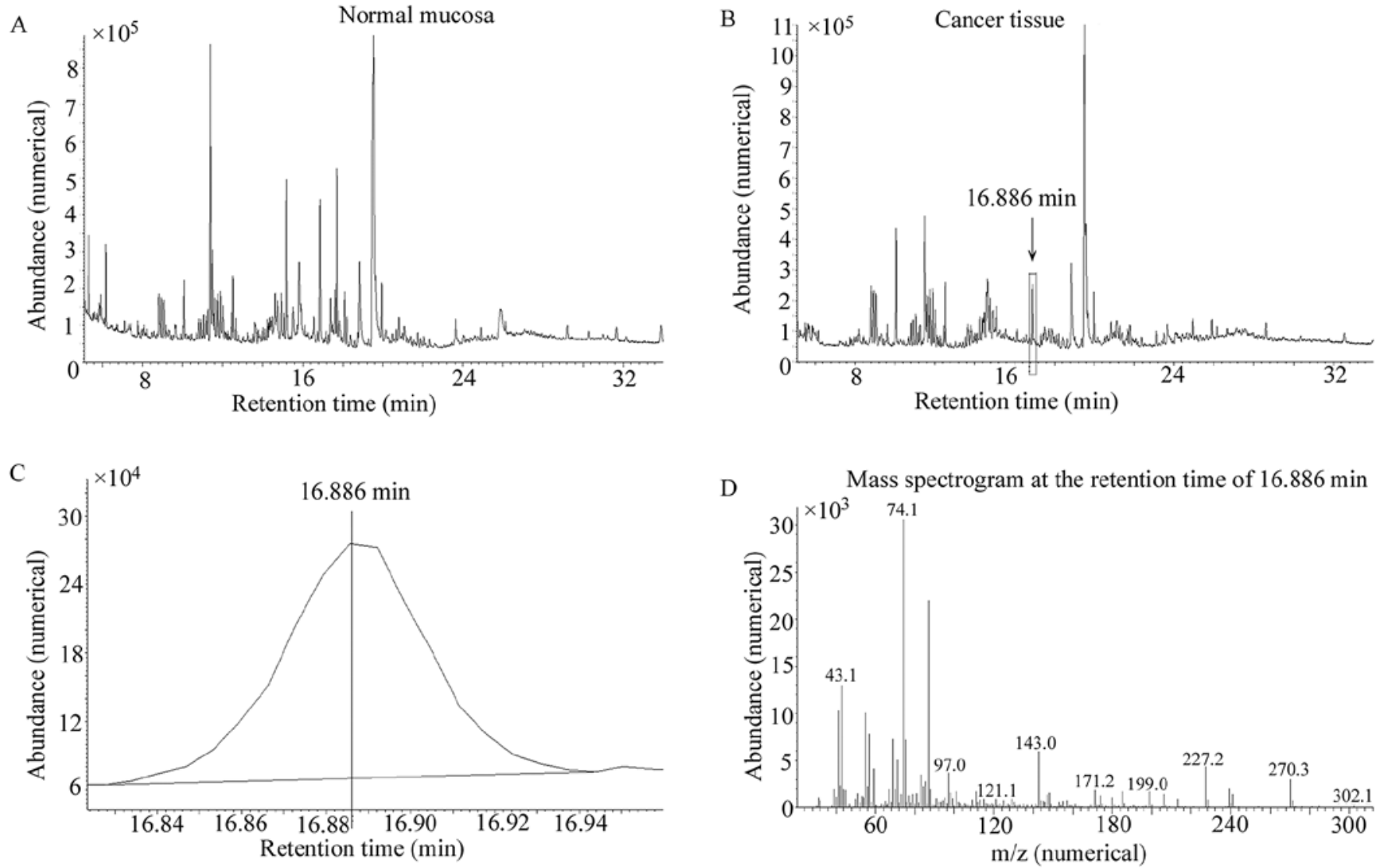

Figure 1. Typical GC/MS total ion current (TIC) chromatograms. (A and B) Within one TIC chromatogram, over 100 signals were commonly detected in a single normal gastric mucosa or tumor tissue sample. (C) A base peak at the retention time of 16.886 (min) was extracted and magnified. (D) Mass spectrogram at the peak summit was selected, and compounds were identified via mass spectral match with National Institute of Standards and Technology library. The $\mathrm{m} / \mathrm{z}$ values with the first three highest abundances of the fragmentation patterns were 74.1, 43.1 and 143.0.

\section{Results}

GC/MS metabolic profiling and OPLS-DA modeling. Typical GC/MS TIC chromatograms of the tissue samples from tumor and normal mucosa are illustrated in Fig. 1A and B. Within one TIC chromatogram, over 100 signals were consistently detected in an individual sample. However, some of these peaks were not consistently noted in other sets of samples, were too low in concentration, or were of poor spectral quality to be confirmed as metabolites, and thus were not investigated further. As displayed in Table II, a total of 41 compounds, such as amino acids, organic acids, carbohydrates, fatty acids and steroids, among the low-molecular-weight endogenous metabolites were detected sequentially in the tumor and normal mucosal tissues.

For the metabolic profile, the OPLS-DA model demonstrated satisfactory modeling and predictive abilities using one predictive component and three orthogonal components $\left(\mathrm{R}^{2}\right.$ Ycum $=0.607, \mathrm{Q}^{2}$ cum $\left.=0.486\right)$, achieving a distinct separation trend between the metabolite profiles of the two groups as shown in Fig. 2. However, a separate OPLS-DA model failed to discriminate normal mucosa and different pathological stages (I-IV) of GC tissues.

Analysis of highlighted metabolites. For OPLS-DA modeling, to identify which variables account for such a significant separation, VIP statistics was initially used to pre-select variables. As shown in Table II, according to the criterion for VIP statistics (VIP $>1$ ), a total of 20 variables were obtained which contributed most toward discriminating the metabolic profiles between the two classes. Wilcoxon test $(\mathrm{p}<0.05)$ was used to investigate differences in the biomolecules in the tumor and normal mucosal samples. A total of 15 metabolites including carbohydrates, amino acids, fatty acids and steroids was detected with significant differences as displayed in Table II. Intriguingly, 7 of the metabolites detected were found to be up-regulated in cancer tissues, while 8 were down-regulated. Octadecanoic acid was the metabolite found to be increased to a greater extent in tumor tissues, showing the greatest fold change (FC, 1.69) while squalene was the metabolite most extensively depleted (FC, -2.73). Meanwhile 12 metabolites were identified using both VIP values (VIP $>1$ ) and the Wilcoxon test $(\mathrm{p}<0.05)$.

\section{Discussion}

The use of GC/MS in the metabolic profiling of esophageal cancer (19), colorectal cancer $(10,26,27,35)$, liver cancer (16), bladder cancer (30) and ovarian cancer (32) has been reported, while few studies have investigated the metabolic profiles of GC tissue. This study explored the metabolic fingerprinting of GC tumor specimens and their matched normal mucosa. A 
Table II. Differential tissue metabolites derived from GC/MS chromatograms.

\begin{tabular}{|c|c|c|c|c|c|c|c|c|c|c|}
\hline Key & $\begin{array}{l}\text { Retention } \\
\text { time }\end{array}$ & $\begin{array}{c}\mathrm{m} / \mathrm{z} \\
\text { no. } 1^{\mathrm{a}}\end{array}$ & $\begin{array}{c}\mathrm{m} / \mathrm{z} \\
\mathrm{no} .2^{\mathrm{a}}\end{array}$ & $\begin{array}{c}\mathrm{m} / \mathrm{z} \\
\mathrm{no} .3^{\mathrm{a}}\end{array}$ & $\begin{array}{l}\text { Match } \\
(\%)^{\mathrm{a}}\end{array}$ & Compound $^{\mathrm{b}}$ & $\begin{array}{l}\text { Chemical } \\
\text { class }\end{array}$ & $\begin{array}{c}\text { VIP } \\
\text { value }^{c}\end{array}$ & P-value ${ }^{d}$ & $\mathrm{FC}^{\mathrm{e}}$ \\
\hline 1 & 5.663 & 69.0 & 44.0 & 207.0 & 80 & Glycine & Amino acid & 1.03428 & 0.242 & 1.46 \\
\hline 2 & 6.128 & 80.1 & 51.0 & 108.0 & 91 & $\beta$-alanine & Amino acid & 0.34774 & 0.897 & -1.06 \\
\hline 3 & 7.836 & 133.1 & 69.0 & 44.0 & 94 & $\beta$-hydroxypyruvic acid & Organic acid & 0.52023 & 0.545 & -1.1 \\
\hline 4 & 7.965 & 69.0 & 44.0 & 113.0 & 83 & $\beta$-mercaptoethylamine & Amino acid & 1.12688 & 0.220 & 1.31 \\
\hline 5 & 8.056 & 6.0 & 43.0 & 113.0 & 84 & Arginine & Amino acid & 0.01426 & 0.955 & 1.10 \\
\hline 6 & 8.936 & 69.1 & 43.1 & 179.1 & 81 & Propanedioic acid & Organic acid & 0.10248 & 0.990 & -1.02 \\
\hline 7 & 9.220 & 69.0 & 43.0 & 91.0 & 85 & Phenylalanine & Amino acid & 1.31881 & 0.305 & 1.59 \\
\hline 8 & 9.641 & 73.1 & 44.0 & 189.1 & 89 & 1-Leucine & Amino acid & 1.61764 & 0.054 & -1.60 \\
\hline 9 & 10.715 & 43.1 & 71.1 & 155.2 & 84 & Maleamic acid & Organic acid & 0.12943 & 0.657 & -1.04 \\
\hline 10 & 11.588 & 69.1 & 43.1 & 191.1 & 85 & Succinic acid & Organic acid & 0.20359 & 0.773 & 1.04 \\
\hline 11 & 12.118 & 43.1 & 57.1 & 71.1 & 90 & Fumaric acid & Organic acid & 1.80591 & 0.043 & 1.31 \\
\hline 12 & 12.519 & 71.0 & 43.0 & 111.0 & 86 & Valeric acid & Organic acid & 1.28267 & 0.035 & 1.08 \\
\hline 13 & 13.820 & 147.0 & 206.1 & 69.0 & 94 & L-(+)-Lactic acid & Organic acid & 0.39049 & 0.563 & 1.17 \\
\hline 14 & 14.589 & 206.0 & 174.0 & 69.0 & 89 & $\alpha$-ketoglutaric acid & Organic acid & 1.22431 & 0.022 & 1.68 \\
\hline 15 & 14.641 & 69.1 & 43.1 & 206.1 & 99 & Tetradecanoic acid & Fatty acid & 0.40702 & 0.371 & -1.07 \\
\hline 16 & 14.758 & 69.1 & 43.1 & 147.0 & 92 & Dodecanamide & Fatty acid & 0.48839 & 0.642 & -1.07 \\
\hline 17 & 15.100 & 69.0 & 43.0 & 206.1 & 86 & d-Glucose & Carbohydrates & 1.06983 & 0.344 & 1.22 \\
\hline 18 & 15.269 & 71.1 & 43.1 & 147.1 & 96 & 1-Pantoyl lactone & Others & 0.15639 & 0.466 & -1.05 \\
\hline 19 & 16.129 & 149.0 & 75.0 & 206.0 & 81 & Xylonic acid & Fatty acid & 0.80958 & 0.043 & 1.06 \\
\hline 20 & 16.588 & 69.0 & 43.1 & 147.1 & 87 & 9-Hexadecenoic acid, (Z)- & Fatty acid & 1.55650 & 0.015 & -1.47 \\
\hline 21 & 16.750 & 69.0 & 43.0 & 206.0 & 82 & 3-Hydroxybutanoic acid & Organic acid & 1.47481 & 0.047 & -1.55 \\
\hline 22 & 16.886 & 74.1 & 43.1 & 143.0 & 95 & Hexadecanoic acid & Fatty acid & 1.27088 & 0.042 & -1.16 \\
\hline 23 & 17.513 & 149.0 & 57.1 & 85.1 & 99 & Pentadecanoic acid & Fatty acid & 1.20398 & 0.202 & 1.25 \\
\hline 24 & 17.733 & 69.1 & 43.1 & 111.1 & 92 & Octadec-9-enoic acid & Fatty acid & 0.70704 & 0.428 & -1.22 \\
\hline 25 & 18.082 & 69.1 & 43.1 & 91.1 & 88 & Ricinoleic acid & Fatty acid & 0.53291 & 0.642 & -1.11 \\
\hline 26 & 18.639 & 69.1 & 43.1 & 111.1 & 84 & Non-ahexacontanoic acid & Fatty acid & 1.16535 & 0.335 & 1.15 \\
\hline 27 & 18.839 & 147.1 & 238.1 & 206.1 & 90 & $2,4(1 \mathrm{H}, 3 \mathrm{H})$-pteridinedione & Others & 0.21137 & 0.475 & 1.02 \\
\hline 28 & 19.519 & 238.1 & 147.1 & 206.1 & 83 & Benzenepropanoic acid & Organic acid & 1.20360 & 0.010 & 1.17 \\
\hline 29 & 19.978 & 74.0 & 43.1 & 143.1 & 99 & Heptadecanoic acid & Fatty acid & 0.48759 & 0.853 & 1.03 \\
\hline 30 & 20.715 & 75.1 & 43.0 & 105.1 & 98 & Octadecanoic acid & Fatty acid & 1.53003 & 0.032 & 1.69 \\
\hline 31 & 20.838 & 59.1 & 85.0 & 117.1 & 93 & Hexadecanamide & $\begin{array}{l}\text { Fatty acid } \\
\text { amide }\end{array}$ & 0.46131 & 0.382 & 1.05 \\
\hline 32 & 21.601 & 69.0 & 43.1 & 97.0 & 80 & Decanoic acid & Fatty acid & 1.09565 & 0.067 & 1.11 \\
\hline 33 & 21.918 & 69.1 & 43.1 & 111.0 & 83 & cis-Vaccenic acid & Fatty acid & 0.65492 & 0.038 & -1.11 \\
\hline 34 & 22.087 & 69.0 & 43.0 & 281.0 & 99 & Arachidonic acid & Fatty acid & 1.05918 & 0.011 & -1.44 \\
\hline 35 & 23.109 & 207.0 & 69.0 & 239.1 & 95 & Eicosanoic acid & Fatty acid & 0.35842 & 0.316 & 1.14 \\
\hline 36 & 23.477 & 239.1 & 57.1 & 85.1 & 93 & $\begin{array}{l}\text { 1-Phenanthrene- } \\
\text { carboxylic acid }\end{array}$ & Organic acid & 1.68511 & 0.031 & 1.60 \\
\hline 37 & 23.671 & 43.0 & 69.0 & 207.1 & 97 & 9-Octadecenamide, $(\mathrm{Z})$ - & $\begin{array}{l}\text { Fatty acid } \\
\text { amide }\end{array}$ & 0.95007 & 0.041 & -1.23 \\
\hline 38 & 24.053 & 59.0 & 104.9 & 207.0 & 87 & Octadecanamide & $\begin{array}{l}\text { Fatty acid } \\
\text { amide }\end{array}$ & 0.55837 & 0.416 & 1.07 \\
\hline 39 & 24.959 & 91.1 & 57.1 & 207.0 & 80 & Ascorbyl palmitate & Fatty acid & 1.04819 & 0.376 & 1.10 \\
\hline 40 & 26.019 & 43.0 & 105.1 & 386.4 & 86 & Cholesterol & Steroids & 0.76534 & 0.545 & 1.27 \\
\hline 41 & 30.347 & 207.0 & 281 & 135.1 & 83 & Squalene & Others & 1.53295 & 0.015 & -2.73 \\
\hline
\end{tabular}

${ }^{a}$ The first three fragment-ion $\mathrm{m} / \mathrm{z}$ values with the highest abundance within each fragmentation pattern and the matching percentage with the NIST library were listed. ${ }^{b}$ Metabolites were identified using an available standard reference or NIST library databases. ${ }^{c}$ Variable importance in the projection (VIP) was obtained from OPLS-DA with a threshold of 1.0. ${ }^{\mathrm{d} P}$-value and fold change (FC) were calculated using the nonparametric Wilcoxon test (significance at $\mathrm{p}<0.05$ ). ${ }^{\mathrm{e}} \mathrm{FC}$ with a positive value indicates a relatively higher concentration present in the tumor specimen, while a negative value means a relatively lower concentration as compared to the normal mucosal sample. 


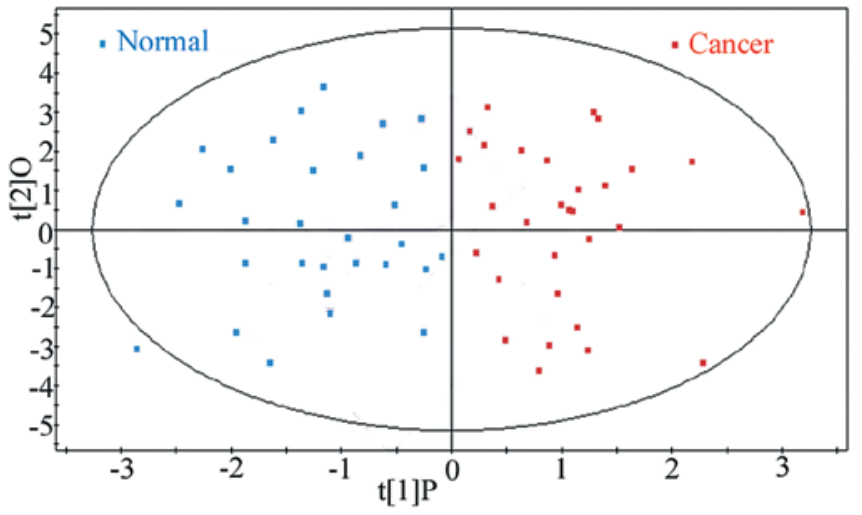

Figure 2. Establishment of the OPLS-DA model. The figure illustrates the score plot of OPLS-DA modeling for tissue metabolomic data. The normal mucosa group tended to cluster to the left region, while the GC tissue group generally clustered to the right area. The OPLS-DA models demonstrated satisfactory modeling and achieved a fairly distinct separation between the metabolite profiles of the two groups.

multivariate statistical method, OPLS-DA, coupled with a univariate statistical method, Wilcoxon test, were adopted in this study, and selection of highlighted metabolites was performed using two parameters, a threshold of 1.0 by VIP and a p-value set to 0.05 , to identify variables with biological significance as end-points of altered interdependent biochemical pathways.

OPLS-DA modeling for the observation of overall separation trends between cancer and non-cancer cohorts has been adopted in human colorectal and bladder cancer $(26,27,30,35)$. Previous studies indicate that the supervised model is a robust model for discrimination. The OPLS-DA model derived from our present GC/MS metabolic analysis showed satisfactory and adequate separations between the $\mathrm{GC}$ and non-GC cohorts, indicating the diagnostic potential of GC metabolic fingerprinting. It has been reported that the unsupervised PCA model also separates GC tissues from normal mucosae (36). Our attempt to stratify GC patients according to TNM staging (stage I-IV) was not successful. Thus, we infer that metabolic phenotypic perturbations may not be directly associated with different GC pathological stages in GC tumor or serum metabolic profiles. We also conceive that GC/MS analysis may not be sensitive enough to discriminate between different TNM stages.

The GC/MS analysis also revealed several marker metabolites related to the metabolic disturbance in GC tissue as shown in Fig. 3. These marker metabolites include carbohydrates, amino acids, fatty acids, cholesterol and other low-molecularweight organic acids, which suggest that they play certain roles in the onset of GC. First of all, a significant increase in several components of aerobic glycolytic pathways, such as fumaric acid and $\alpha$-ketoglutaric acid was noted in the tumor tissues. This enhanced carbohydrate consumption in tumors suggests there may be a switch to the Warburg effect (37). It has been reported that cancer cells predominantly produce energy by glycolysis rather than oxidative phosphorylation via the tricarboxylic acid (TCA) cycle (38). Promotion of glycolysis and disruption of the TCA cycle were also noted in invasive ovarian carcinoma (32), liver cancer (39) and colorectal cancer (40). Abnormal metabolism of carbohydrates may be associated with tumor growth with a large energy requirement needed for aggressive cell proliferation. Moreover, enhanced glucose uptake appears to be a metabolic feature of cancer cells, although the metabolic mechanisms linking increased aerobic glycolysis to the development of cancer still remain controversial (41).

Another notable feature of the present analysis was the alteration of fatty acid metabolism in GC tissues. The level of

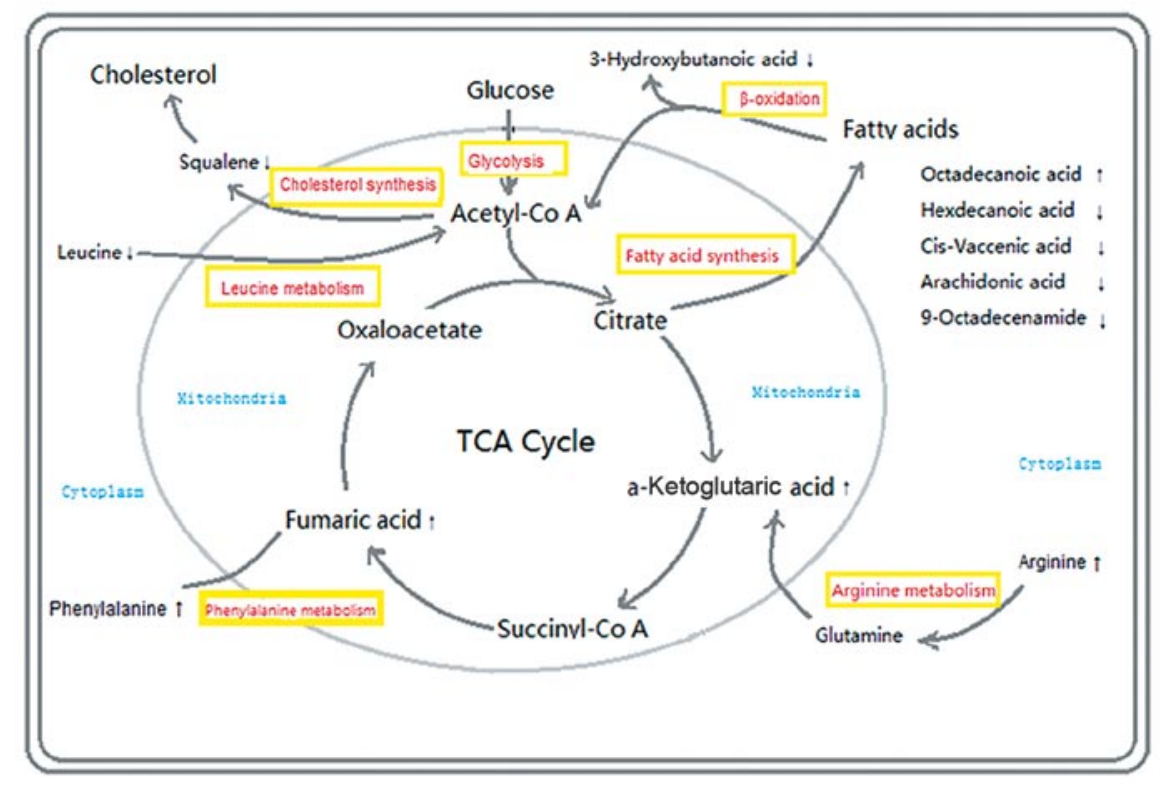

Figure 3. Alteration of the metabolic profile in GC tissues. The model illustrates various prominent aspects of metabolic alteration from normal mucosa to the tumor. The increased level of fumaric acid and $\alpha$-ketoglutaric acid indicates the enhanced glycolytic pathway. Altered fatty acids and cholesterol levels in tumor cells suggest the dysfunction of lipid and cholesterol metabolism. Altered amino acids such as leucine, phenylalanine and arginine take part in the TCA cycle in some manner, and this feature may be noted as an indication for the perturbation of amino acid metabolism. 
a wide spectrum of fatty acids particularly unsaturated fatty acid such as 9-hexadecenoic acid, cis-vaccenic acid and arachidonic acid was found to be significantly decreased in tumor tissues when compared to the adjacent mucosa tissues. The level of hexadecanoic acid was also found to be significantly decreased in tumor tissues while the level of octadecanoic acid was found to be elevated. The lower level of 3-hydroxybutanoic acid, the end-point product of fatty acids $\beta$-oxidation, was also detected in the tumor tissues. Fatty acid metabolic alterations have been observed in several other cancer types. Saturated fatty acids were found significantly increased in lung cancer tissues, while unsaturated fatty acids were decreased in lung cancer tissues (42). Comparatively, saturated fatty acids such as non-adecanoic acid, stearic acid and heptadecanoic acid were found to be significantly decreased in invasive ovarian carcinomas when compared to ovarian border-line tumors (32). As reported, the level of fatty acids from $\mathrm{C}-12$ to $\mathrm{C}-22$ together with various glycerols was found to be significantly elevated in colorectal polyps and colorectal cancer tissues as compared to adjacent mucosa tissues (27). Fatty acids are used for both structural purposes and energy production, such as $\beta$-oxidation. These findings indicate the dysfunction of cell membrane synthesis and fatty acid $\beta$-oxidation metabolism, which is confirmed by previous research (43), and different fatty acids may play their respective role in the process of lipid metabolism.

Although the decrease in cholesterol was not as significant, a significant decrease in squalene, the intermediate product of cholesterol synthesis, revealed that the synthesis of cholesterol was inhibited in GC cells. It has been reported that the genes encoding two key enzymes in cholesterol synthesis are located in chromosome $5(44,45)$, which has been shown to be the location of one of the genes involved in the adenoma-carcinoma sequence $(46,47)$. It is possible that deletions in genes on chromosome 5 lead both to decreased cholesterol synthesis and to the progression of adenoma to cancer. The present study suggests that a metabolic variation in cholesterol levels may be related to $\mathrm{GC}$ tumorigenesis.

Perturbations of amino acid metabolism in GC tissues were also noted in the present study. A lower concentration of leucine was detected with significant difference, while glycine (FC, 1.46), phenylalanine (FC, 1.59) and arginine (FC, 1.1) were detected with elevated concentrations although no statistical significances were confirmed. Leucine is one type of ketogenic amino acids while glycine and arginine belong to the glucogenic amino acids. Phenylalanine is one of the glucogenic and ketogenic amino acids. Their intermediate products such as acetyl coenzyme A, $\alpha$-ketoglutaric acid and fumaric acid participate in the TCA cycle. As reported, branched chain amino acids have been found to be increased in hepatocellular carcinoma due to increased glycolysis $(48,49)$. The present findings may indicate the involvement of glycolysis, and we believe that GC cells selectively utilize amino acids in pathophysiologic metabolism. Several essential and non-essential amino acids including phenylalanine, proline, methionine, lysine and glutamate have been found stepwisely and significantly increased from colonic mucosa through polyps to colorectal cancer, suggesting that an increase in protein synthesis is essential for rapidly growing tumor cells (27). The present results also indicate that abnormal protein synthesis occurs in
GC cells, and the altered amino acids are phenotypic markers for abnormal protein synthesis.

In summary, utilizing the highly sensitive metabolomic technique, we identified signature metabolic shifts in GC tissues with multivariate and univariate statistical significance. The unique metabolic profiles generated by GC/MS revealed metabolic perturbations in GC cells. The results of this study also indicate the potential of this efficient robust profiling approach for detection of GC. In light of this, many differential endogenous biomarkers as well as macromolecular metabolites warrant further investigation and are yet to be identified. The next step in our study will include the identification of key differential biomarkers and the investigatin of the metabolic shift of various macromolecular metabolites.

\section{Acknowledgements}

This study was financially supported by the Science Technology Development Program of Guangdong, China (no. 2008B030301105). We are grateful to the National Engineering Research Center of Genetic Medicine, Ji Nan University, for the technical support. We thank the patients for taking part in this study.

\section{References}

1. Parkin DM, Bray F, Ferlay J and Pisani P: Global cancer statistics, 2002. CA Cancer J Clin 55: 74-108, 2005.

2. Crew KD and Neugut AI: Epidemiology of gastric cancer. World J Gastroenterol 12: 354-362, 2006.

3. Yasui W, Oue N, Aung PP, Matsumura S, Shutoh M and Nakayama H: Molecular-pathological prognostic factors of gastric cancer: a review. Gastric Cancer 8: 86-94, 2005.

4. Macdonald JS: Gastric cancer - new therapeutic options. N Engl J Med 355: 76-77, 2006

5. Cunningham D and Chua YJ: East meets west in the treatment of gastric cancer. N Engl J Med 357: 1863-1865, 2007.

6. Väänänen H, Vauhkonen M, Helske T, et al: Non-endoscopic diagnosis of atrophic gastritis with a blood test. Correlation between gastric histology and serum levels of gastrin-17 and pepsinogen I: a multicentre study. Eur J Gastroenterol Hepatol 15: 885-891, 2003.

7. Kikuchi S, Kurosawa M, Sakiyama T, Tenjin H, Miki K, Wada O and Inaba Y: Long-term effect of Helicobacter pylori infection on serum pepsinogens. Jpn J Cancer Res 91: 471-476, 2000.

8. Kitahara F, Kobayashi K, Sato T, Kojima Y, Araki T and Fujino MA: Accuracy of screening for gastric cancer using serum pepsinogen concentrations. Gut 44: 693-697, 1999.

9. Miki K, Morita M, Sasajima M, Hoshina R, Kanda E and Urita Y: Usefulness of gastric cancer screening using the serum pepsinogen test method. Am J Gastroenterol 98: 735-739, 2003.

10. Mal M, Koh PK, Cheah PY and Chan EC: Development and validation of a gas chromatography/mass spectrometry method for the metabolic profiling of human colon tissue. Rapid Commun Mass Spectrom 23: 487-494, 2009.

11. Nicholson JK, Connelly J,Lindon JC and Holmes E: Metabonomics: a platform for studying drug toxicity and gene function. Nat Rev Drug Discov 1: 153-161, 2002.

12. Holmes E, Wilson ID and Nicholson JK: Metabolic phenotyping in health and disease. Cell 134: 714-717, 2008.

13. Deng C, Li N and Zhang X: Development of headspace solidphase microextraction with on-fiber derivatization for determination of hexanal and heptanal in human blood. J Chromatogr B Analyt Technol Biomed Life Sci 813: 47-52, 2004.

14. Wikoff WR, Gangoiti JA, Barshop BA and Siuzdak G: Metabolomics identifies perturbations in human disorders of propionate metabolism. Clin Chem 53: 2169-2176, 2007.

15. Claudino WM, Quattrone A, Biganzoli L, Pestrin M, Bertini I and Di Leo A: Metabolomics: available results, current research projects in breast cancer, and future applications. J Clin Oncol 25: 2840-2846, 2007. 
16. Xue R, Lin Z, Deng C, Dong L, Liu T, Wang J and Shen X: A serum metabolomic investigation on hepatocellular carcinoma patients by chemical derivatization followed by gas chromatography/mass spectrometry. Rapid Commun Mass Spectrom 22: 3061-3068, 2008.

17. Bogdanov M, Matson WR, Wang L, Matson T, SaundersPullman R, Bressman SS and Flint Beal M: Metabolomic profiling to develop blood biomarkers for Parkinson's disease. Brain 131: 389-396, 2008.

18. Chen J, Zhao X, Fritsche J, et al: Practical approach for the identification and isomer elucidation of biomarkers detected in a metabonomic study for the discovery of individuals at risk for diabetes by integrating the chromatographic and mass spectrometric information. Anal Chem 80: 1280-1289, 2008.

19. Wu H, Xue R, Lu C, et al: Metabolomic study for diagnostic model of oesophageal cancer using gas chromatography/mass spectrometry. J Chromatogr B Analyt Technol Biomed Life Sci 877: 3111-3117, 2009.

20. Deng C, Zhang $X$ and Li N: Investigation of volatile biomarkers in lung cancer blood using solid-phase microextraction and capillary gas chromatography-mass spectrometry. J Chromatogr B Analyt Technol Biomed Life Sci 808: 269-277, 2004.

21. Griffin JL and Shockcor JP: Metabolic profiles of cancer cells. Nat Rev Cancer 4: 551-561, 2004.

22. Jonsson P, Gullberg J, Nordström A, Kusano M, Kowalczyk M, Sjöström M and Moritz T: A strategy for identifying differences in large series of metabolomic samples analyzed by GC/MS Anal Chem 76: 1738-1745, 2004.

23. Deng C, Zhang J, Yu X, Zhang W and Zhang X: Determination of acetone in human breath by gas chromatography-mass spectrometry and solid-phase microextraction with on-fiber derivatization. J Chromatogr B Analyt Technol Biomed Life Sci 810: 269-275, 2004.

24. Jonsson P, Johansson AI, Gullberg J, et al: High-throughput data analysis for detecting and identifying differences between samples in GC/MS-based metabolomic analyses. Anal Chem 77: 5635-5642, 2005.

25. Sobin LH and Fleming ID: TNM Classification of Malignant Tumors, 5th edition (1997). Union Internationale Contre le Cancer and the American Joint Committee on Cancer. Cancer 80: 1803-1804, 1997.

26. Qiu Y, Cai G, Su M, et al: Serum metabolite profiling of human colorectal cancer using GC-TOFMS and UPLC-QTOFMS. J Proteome Res 8: 4844-4850, 2009.

27. Ong ES, Zou L, Li S, Cheah PY, Eu KW and Ong CN: Metabolic profiling in colorectal cancer reveals signature metabolic shifts during tumorigenesis. Mol Cell Proteomics. In Press.

28. Ong ES, Chor CF, Zou L and Ong CN: A multi-analytical approach for metabolomic profiling of zebrafish (Danio rerio) livers. Mol Biosyst 5: 288-298, 2009.

29. Law WS, Huang PY, Ong ES, Sethi SK, Saw S, Ong CN and Li SF: Combination of $1 \mathrm{H}$ nuclear magnetic resonance spectroscopy and liquid chromatography/mass spectrometry with pattern recognition techniques for evaluation of metabolic profile associated with albuminuria. J Proteome Res 8: 1828-1837, 2009.

30. Pasikanti KK, Esuvaranathan K, Ho PC, et al: Non-invasive urinary metabonomic diagnosis of human bladder cancer. J Proteome Res 9: 2988-2995, 2010.

31. Görgényi M, Fekete ZA, van Langenhove $H$ and Dewulf J: Temperature dependence of the Kováts retention index. Convex or concave curves. J Chromatogr A 1206: 178-185, 2008.
32. Denkert C, Budczies J, Kind T, et al: Mass spectrometry-based metabolic profiling reveals different metabolite patterns in invasive ovarian carcinomas and ovarian borderline tumors. Cancer Res 66: 10795-10804, 2006.

33. Trygg J, Holmes E and Lundstedt T: Chemometrics in metabonomics. J Proteome Res 6: 469-479, 2007.

34. Bylesjö M, Eriksson D, Kusano M, Moritz T and Trygg J: Data integration in plant biology: the O2PLS method for combined modeling of transcript and metabolite data. Plant J 52: 1181-1191, 2007.

35. Chan EC, Koh PK, Mal M, et al: Metabolic profiling of human colorectal cancer using high-resolution magic angle spinning nuclear magnetic resonance (HR-MAS NMR) spectroscopy and gas chromatography mass spectrometry (GC/MS). J Proteome Res 8: 352-361, 2009.

36. Wu H, Xue R, Tang Z, et al: Metabolomic investigation of gastric cancer tissue using gas chromatography/mass spectrometry. Anal Bioanal Chem 396: 1385-1395, 2010.

37. Warburg O: On the origin of cancer cells. Science 123: 309-314, 1956.

38. Hirayama A, Kami K, Sugimoto M, et al: Quantitative metabolome profiling of colon and stomach cancer microenvironment by capillary electrophoresis time-of-flight mass spectrometry. Cancer Res 69: 4918-4925, 2009.

39. Wang Y, Bollard ME, Keun H, et al: Spectral editing and pattern recognition methods applied to high-resolution magic-angle spinning $1 \mathrm{H}$ nuclear magnetic resonance spectroscopy of liver tissues. Anal Biochem 323: 26-32, 2003.

40. Yang Y, Li C, Nie X, et al: Metabonomic studies of human hepatocellular carcinoma using high-resolution magic-angle spinning 1H NMR spectroscopy in conjunction with multivariate data analysis. J Proteome Res 6: 2605-2614, 2007.

41. Zambell KL, Fitch MD and Fleming SE: Acetate and butyrate are the major substrates for de novo lipogenesis in rat colonic epithelial cells. J Nutr 133: 3509-3515, 2003.

42. Cha D, Liu M, Zeng Z, Cheng D and Zhan G: Analysis of fatty acids in lung tissues using gas chromatography-mass spectrometry preceded by derivatization-solid-phase microextraction with a novel fiber. Anal Chim Acta 572: 47-54, 2006.

43. Mazzanti R, Solazzo M,Fantappié O, et al: Differential expression proteomics of human colon cancer. Am J Physiol Gastrointest Liver Physiol 290: G1329-G1338, 2006.

44. Leonard S, Arbogast D, Geyer D, Jones C and Sinensky M: Localization of the gene encoding 3-hydroxy-3-methylglutarylcoenzyme A synthase to human chromosome 5. Proc Natl Acad Sci USA 83: 2187-2189, 1986.

45. Mohandas T, Heinzmann C, Sparkes RS, Wasmuth J, Edwards P and Lusis AJ: Assignment of human 3-hydroxy-3-methylglutaryl coenzyme A reductase gene to $\mathrm{q} 13 \rightarrow \mathrm{q} 23$ region of chromosome 5. Somat Cell Mol Genet 12: 89-94, 1986.

46. Solomon E, Voss R, Hall V, et al: Chromosome 5 allele loss in human colorectal carcinomas. Nature 328: 616-619, 1987.

47. Bodmer WF, Bailey CJ, Bodmer J, et al: Localization of the gene for familial adenomatous polyposis on chromosome 5 . Nature 328: 614-616, 1987.

48. Xue R, Dong L, Wu H, Liu T, Wang J and Shen X: Gas chromatography/mass spectrometry screening of serum metabolomic biomarkers in hepatitis B virus infected cirrhosis patients. Clin Chem Lab Med 47: 305-310, 2009.

49. Taniguchi K, Nonami T, Nakao A, et al: The valine catabolic pathway in human liver: effect of cirrhosis on enzyme activities. Hepatology 24: 1395-1398, 1996. 